\title{
Modified Salmonella toxin combats Alzheimer disease in mouse model
}

A compound derived from the lipopolysaccharide (LPS) of Salmonella minnesota reduces plaque burden and improves cognitive function in a mouse model of Alzheimer disease (AD), a new study has shown. The research could help to address the growing unmet need for a disease-modifying treatment for AD.

Previous studies had shown that Toll-like receptors (TLRs) expressed on microglia bind to amyloid- $\beta(A \beta)$ - the protein species that is thought to have a

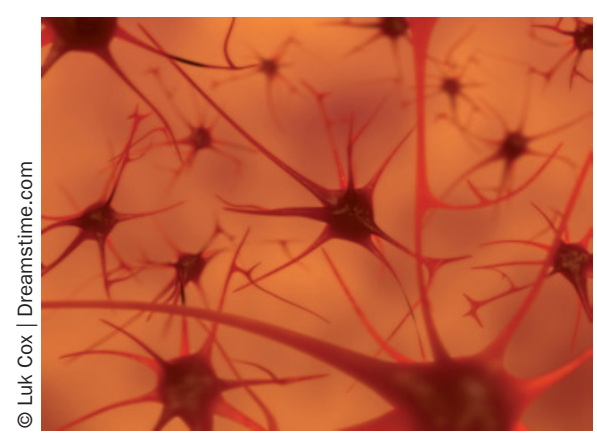

key role in $\mathrm{AD}$ pathology_suggesting the possibility to harness the innate immune system to clear $A \beta$ through stimulation of these receptors. Although LPS stimulates TLR4, the therapeutic potential of this molecule is limited by its inflammatory properties. In the current study, Serge Rivest and colleagues investigated whether a detoxified form of LPS, called monophosphoryl lipid A (MPL), could be used to avoid the adverse effects of LPS.

The researchers found that administration of MPL to mice via intraperitoneal injection led to increased serum levels of inflammatory cytokines, but to a considerably lesser extent than with LPS injection. In addition, in vitro studies showed that MPL stimulated uptake of $A \beta$ by microglia. Together, these findings suggested the potential of MPL to increase $A \beta$ clearance without triggering an adverse immune response.

To directly test this possibility, Rivest et al. administered the compound to an $\mathrm{AD}$ mouse model once per week for 12 weeks. Treated mice performed better in a $\mathrm{T}$ water maze than did PBS-treated mice, indicating that MPL therapy improved cognitive function. Moreover, the compound significantly reduced the number and size of $A \beta$ deposits, as well as the amount of soluble $A \beta$ in the brain.

"This new TLR4 ligand is very powerful to clear $\mathrm{A} \beta$ and improve cognitive functions," says Rivest. "We hope that this will pave the way to a safe and effective immunotherapy for AD." MPL is already included in vaccine formulations for humans, which could help to accelerate its translation to the $\mathrm{AD}$ clinic.

Katie Kingwell

Original article Michaud, J.-P. et al. Toll-like receptor 4 stimulation with the detoxified ligand monophosphoryl lipid A improves Alzheimer's disease-related pathology. Proc. Natl Acad. Sci. USA 110, 1941-1946 (2013) 